\title{
Propiedades nutritivas y saludables de algas marinas y su potencialidad como ingrediente funcional
}

\section{Nutritional and health properties of seaweeds and its potential as a functional ingredient}

\begin{abstract}
Marine algae (seaweeds) have been consumed in Asia since ancient times, while in Western countries the main use of seaweeds has been as sources of gelling and colloidal agents for food, pharmaceutical and cosmetic industry. Seaweed is a rich source of nutrients such as proteins, vitamins, minerals and dietary fiber. Seaweed dietary fibers are particularly rich in the soluble fractions. Compared to the terrestrial vegetables, seaweed is rich in some health-promoting molecules and materials such as w-3 fatty acids and bioactive molecules. The secondary metabolites synthesized by seaweeds have shown antioxidant, antiinflammatory, anticancer and antidiabetic activity. Therefore, seaweeds can be considered as very interesting natural sources containing new compounds with numerous biological activities that could be used as functional ingredients in many industrial applications such as functional food.
\end{abstract}

Key words: Seaweeds, dietary fibre, polyphenols, pigments.

\section{INTRODUCCIÓN}

Las algas son organismos autótrofos de estructura simple, con escasa o nula diferenciación celular y de tejidos complejos por lo que son talofitas. Taxonómicamente se clasifican en tres grupos: Chlorophyta o clorofitas, Phaeophyta o feófitas y Rhodophyta o rodófitas, que corresponden a algas verdes, pardas y rojas respectivamente ya que presentan pigmentos que predominan sobre los otros (1), tal como se aprecia en la tabla 1.

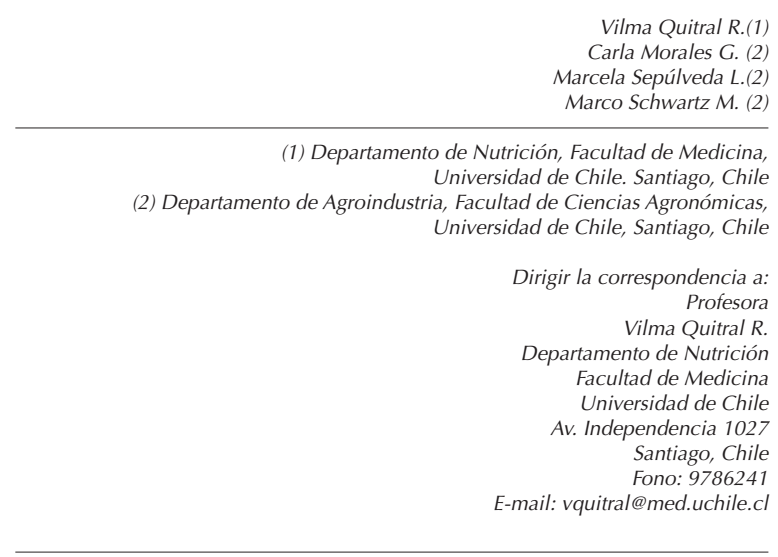

Este trabajo fue recibido el 27 de Julio de 2012 y aceptado para ser publicado el 10 de Diciembre de 2012.

Las algas pardas o Phaeophyta corresponden a un grupo muy grande de algas marinas, en que no se conoce aún el número exacto de especies. Su pigmentación varía de amarillo pardo a pardo oscuro y produce gran cantidad de un mucus protector. Dentro de este grupo de algas, las más conocidas en nuestro país son Macrocystis pyrifera (huiro), Lessonia nigrescens (huiro negro), Durvillaea antarctica (cochayuyo). Las algas rojas o Rhodophyta son el segundo grupo más grande de algas y son las más primitivas, las que se encuentran en diversos

TABLA 1

Clasificación de algas y sus pigmentos (1)

$\begin{array}{llll}\text { Clasificación } & \text { Nombre común } & \text { Pigmentos } & \text { Ejemplos } \\ \text { Clorophyta } & \text { Algas verdes } & \begin{array}{l}\text { Clorofilas a y b, Xantófilas (luteína, violaxantina, } \\ \text { neoxantina y enteroxantina) }\end{array} & \text { Ulva spp., Codium spp. } \\ \text { Phaeophyta } & \text { Algas pardas } & \begin{array}{l}\text { Xantofilas (fucoxantina y flavoxantina) y } \\ \text { Clorofila a y c }\end{array} & \begin{array}{l}\text { Laminaria spp., Lessonia spp., } \\ \text { Sargassum spp., Durvillaea spp. }\end{array} \\ \text { Rhodophyta } & \text { Algas rojas } & \text { Ficoeritrina, ficobilina, clorofilas a y d } & \text { Gracilaria spp., Palmaria spp., } \\ & & & \text { Porphyra spp. }\end{array}$


medios. Las especies Gracilaria (pelillo), Porphyra (luche) y Chondrus crispus (liquén) son algunos ejemplos. Algas verdes o Chlorophyta tienen menor presencia que las algas pardas y rojas. Su pigmentación varía desde amarillo verdoso hasta verde oscuro. Ulva lactuca conocido como ulte o lechuga de mar es la más conocida (2).

Aproximadamente el $66 \%$ de las especies de algas conocidas se usan como alimento, siendo los países asiáticos los mayores consumidores utilizando diversas formas culinarias; en cambio en países occidentales se utilizan principalmente para la extracción de hidrocoloides como agar, carragenina y alginatos (3).

Japón y China son los mayores productores, cultivadores y consumidores de algas en el mundo. El consumo de algas en Japón es de 8.5 g/día, según datos de Korean National Health and Nutrition Survey aunque puede llegar a más de 10 g/día $(4,5)$. Otros países que consumen algas son Escocia, Chile, Filipinas, Malasia, Bali, Corea, Singapur y Sri Lanka (6).

Las algas son un recurso abundante, económico y atractivo para utilizar como ingrediente en alimentos. Aportan nutrientes y compuestos bioactivos, además de tener propiedades tecnológicas que hacen viable su incorporación. La concentración a utilizar debe ser correctamente controlada ya que la calidad sensorial no siempre se ve favorecida, por lo que es un interesante desafío su inclusión en alimentos como un ingrediente funcional.

\section{Valor nutricional de algas}

Desde el punto de vista nutricional, las algas son bajas en calorías, presentan alta concentración de proteínas, fibra dietética, minerales y vitaminas (7-11). La tabla 2 presenta datos de la composición química de distintas algas.

En general, las proteínas de algas son ricas en glicina, arginina, alanina y ácido glutámico; contienen aminoácidos esenciales en niveles comparables a los que indica FAO/OMS como requerimientos, sus aminoácidos limitantes son lisina y cistina $(3,6)$.

En las algas rojas, se encuentra el aminoácido libre taurina, que está presente en la mayoría de los tejidos. Taurina participa en muchos procesos fisiológicos como osmoregulación, inmunomodulación, estabilización de membrana, tiene un rol muy importante en el desarrollo ocular y del sistema nervioso (12-16). Este aminoácido libre es necesario en mayor cantidad durante la infancia que durante la adultez. La fuente principal es la leche materna durante los primeros meses de vida, por lo que se propone fortificar fórmulas infantiles, debido a que la leche de vaca contiene menores concentraciones de taurina que la leche humana (17-19). Los alimentos de origen marino son una mejor fuente de taurina que los alimentos terrestres (16). El aminoácido fosfoserina se encuentra en alta concentración en las algas pardas (20).

Las algas son excelente fuente de vitaminas A, B1, B12, $C, D$ y $E$, riboflavina, niacina, ácido pantoténico y acido fólico (6).

El contenido en minerales en algas es alto, sobre un $36 \%$ de peso seco, dentro de los macrominerales se incluyen sodio, calcio, potasio, cloro, sulfuro y fósforo. Una porción de Ulva lactuca aporta aproximadamente $257 \mathrm{mg}$ de calcio, similar al aporte de queso $(21,22)$. En los microminerales se incluyen el yodo, hierro, zinc, cobre, selenio, molibdeno, flúor, manganeso, boro, níquel y cobalto. Las algas son fuente primaria de yodo, llegando a aportar el requerimiento diario de yodo (150 $\mu \mathrm{g} /$ día) (3).

Las algas presentan una relación $\mathrm{Na} / \mathrm{K}$ baja, del orden de 0.14-0.16, lo que contribuye a disminuir la incidencia de hipertensión, de tal manera que el consumo de algas puede contribuir a balancear la alta relación $\mathrm{Na} / \mathrm{K}$ de la dieta habitual $(21,23)$.

El contenido de lípidos en las algas es bajo (1 a 5\% b.s.), siendo los lípidos neutros y glicolípidos los más abundantes. La proporción de ácidos grasos esenciales en algas es mayor que en plantas terrestres, además sintetizan gran cantidad de ácidos grasos poliinsaturados de cadena larga, en los que destaca el ácido eicosapentaenoico (EPA) y docosahexaenoico (DHA) que pertenecen a la familia de ácidos grasos $\omega-3$. El consumo de estos ácidos grasos se relaciona con disminución del riesgo de padecer enfermedades cardiovasculares, particularmente enfermedad coronaria. El efecto biológico de EPA y DHA es muy extenso y variado, involucra lipoproteínas, presión sanguínea, función cardíaca, función endotelial, reactividad vascular y fisiología cardíaca, así como un efecto antiinflamatorio y antiplaquetario $(24,25)$. Tienen efecto en la disminución del riesgo de enfermedades cardiovasculares (26), disminuyen los niveles de triglicéridos (27), son necesarios durante el embarazo y lactancia para el desarrollo del sistema nerviosos central y retina del infante, además se ha comprobado que su consumo tiene efectos positivos contra la depresión postparto y la depresión bipolar (28). La relación de ácidos grasos $\omega-6: \omega-3$ es muy baja en las algas, lo que es muy beneficioso, ya que relaciones entre 1 y 4 son óptimas (29). La tabla 3 presenta la proporción de EPA y DHA en algas y la relación $\omega-6: \omega-3$.

En general las algas rojas poseen altos contenidos de EPA, ácido palmítico, oleico y araquidónico, en comparación con las algas pardas, que contienen elevadas concentraciones de ácido oleico, linoleico y a-linolénico, pero bajas de EPA.

TABLA 2

Composición química de algas (g/100 g base seca)

\begin{tabular}{|c|c|c|c|c|c|}
\hline Alga & Proteínas & Lípidos & Cenizas & Fibra dietética total & Ref. \\
\hline Grateloupia turuturu & $22.9 \pm 2.0$ & $2.6 \pm 0.1$ & $18.5 \pm 0.6$ & $60.4 \pm 2.3$ & 71 \\
\hline Ulva clathrata & $20.1 \pm 0.1$ & $2.2 \pm 0.1$ & $27.5 \pm 0.2$ & 40.6 & 72 \\
\hline Ulva lactuca & $27.2 \pm 1.1$ & $0.3 \pm 0.0$ & $11.0 \pm 0.1$ & 60.5 & 33 \\
\hline Ulva lactuca & $8.46 \pm 0.01$ & $787 \pm 0.10$ & $19.59 \pm 0.51$ & $54.90 \pm 0.95$ & 73 \\
\hline Durvillaea antarctica (tallo) & $11.6 \pm 0.9$ & $4.3 \pm 0.6$ & $25.7 \pm 2.5$ & - & 33 \\
\hline Laminaria saccharina & $25.70 \pm 0.11$ & $0.79 \pm 0.07$ & $34.78 \pm 0.08$ & - & 74 \\
\hline Hizikia fusiforme & $10.9 \pm 1.0$ & $1.4 \pm 0.1$ & - & $62.3 \pm 0.7$ & 20 \\
\hline
\end{tabular}


Las algas verdes poseen en mayor cantidad ácido linoleico y a-linolénico, palmítico, oleico y $\operatorname{DHA}(30,31)$.

Las algas contienen una alta concentración de hidratos de carbono como polisacáridos estructurales, de almacenamiento y funcionales, con valores de 20 a 70\%. La proporción de fibra dietética es considerable, puede variar de 36 a $60 \%$ de su materia seca (32) siendo muy alta la fibra dietética soluble (aproximadamente 55-70\%) en comparación con vegetales terrestres (3). Por lo tanto las algas no son una buena fuente de hidratos de carbono en términos de biodisponibilidad.

Dawczynski et al. (20) no encontraron diferencias significativas en el contenido de fibra dietética entre algas rojas y pardas, con valores promedio de 48.6 y 43.8 g/100g respectivamente. Ortiz et al. (33) compararon el contenido de fibra dietética de algas Ulva lactuca y Durvillaea antarctica extraídas en Chile, conocidas como "ulte" y "cochayuyo" respectivamente, con valores de frutas y hortalizas, encontrando mayor el contenido de fibra dietética en las algas.

La tabla 4 presenta los valores de fibra dietética de diferentes algas; el promedio calculado para fibra soluble es $24.5 \mathrm{~g} / 100 \mathrm{~g}$ y para fibra insoluble es $21.8 \mathrm{~g} / 100 \mathrm{~g}$; en frutas el promedio es $4.6 \mathrm{~g} / 100 \mathrm{~g}$ y $10.2 \mathrm{~g} / 100 \mathrm{~g}$, mientras que en verduras es $8.9 \mathrm{~g} / 100 \mathrm{~g}$ y $19.5 \mathrm{~g} / 100 \mathrm{~g}$ para fibra soluble e insoluble respectivamente $(34,35)$. Las algas tienen alta proporción de fibra soluble $(7,36)$, que se caracteriza por su capacidad de aumentar la viscosidad, reducir la respuesta glicémica y el colesterol en el plasma (37). La relación F. Soluble/F. Insoluble $(\mathrm{S} / \mathrm{I})$ es mayor en algas que en vegetales terrestres.
2. Compuestos bioactivos presentes en algas

Aparte de sus componentes nutritivos, las algas contienen compuestos bioactivos de alta capacidad antioxidante, como carotenoides y polifenoles $(33,38-42)$. Se han investigado los pigmentos naturales de las algas encontrando actividad antioxidante, anticancerígena, antiinflamatoria (basado principalmente sobre la modulación de función de macrófagos), entre otras (43). La tabla 5 presenta concentración de carotenoides y tocoles en algas.

Dentro de los pigmentos naturales de algas se destaca la fucoxantina, carotenoide que incluye un enlace alénico y 5,6-monoepoxido en su molécula (figura 1). Se encuentra disponible en diferentes especies de algas pardas. La absorción de fucoxantina por el organismo depende de diversos factores como cantidad y tipo de lípidos consumidos, la estabilidad de la matriz en la que se encuentra unida la fucoxantina y factores adicionales como fibra dietética, además de otros que no se encuentran totalmente dilucidados (44).

Diversos autores han demostrado que la fucoxantina de diferentes tipos de algas tiene un efecto antioxidante, anticancerígeno, antiinflamatorio, antiobesidad, neuroprotector, fotoprotector y preventivo de osteoporosis (45-50).

Fucoxantina y fucoxantinol aislados de algas inhiben la diferenciación de preadipocitos 3T3-L1 en adipocitos (51). Los estudios científicos apoyan la hipótesis de que otros carotenoides con grupo alénico y grupo hidroxilo adicional tienen un efecto en la supresión de la diferenciación de adipocitos (43). Estudios realizados en un modelo animal diabético/obeso

TABLA 3

Contenido de lípidos, EPA, DHA y relación $\omega-6: \omega-3$ en algas

\begin{tabular}{|c|c|c|c|c|c|}
\hline Alga & Lípidos g/100 g & EPA (\%) & DHA (\%) & Relación $\omega-6: \omega-3$ & Ref. \\
\hline Ulva lactuca & $0.3 \pm 0.0$ & $1.01 \pm 0.01$ & $0.8 \pm 0.01$ & 1.31 & 33 \\
\hline Durvillaea Antarctica (hojas) & $0.8 \pm 0.1$ & $4.95 \pm 0.11$ & $1.66 \pm 0.02$ & 2.0 & 33 \\
\hline Codium fragile & $1.5 \pm 0.0$ & $2.10 \pm 0.00$ & - & 0.32 & 11 \\
\hline Gracilaria chilensis & $1.3 \pm 0.0$ & $1.30 \pm 0.01$ & - & 3.42 & 11 \\
\hline Macrocystis pyrifera & $0.7 \pm 0.1$ & $0.47 \pm 0.01$ & - & 7.42 & 11 \\
\hline Porphyra sp. (China) & - & $10.4 \pm 7.46$ & No detectable & 1.8 & 20 \\
\hline Undaria pinnatifia & - & $13.2 \pm 0.66$ & No detectable & 0.5 & 20 \\
\hline Laminaria sp. & - & $16.2 \pm 8.9$ & No detectable & 1.3 & 20 \\
\hline Ulva lactuca & $1.27 \pm 0.11$ & $0.87 \pm 0.16$ & $2.15 \pm 0.44$ & 3.0 & 31 \\
\hline
\end{tabular}

TABLA 4

Contenido de fibra dietética y relación F.S./F.I. en algas

\begin{tabular}{|c|c|c|c|c|}
\hline Alga & $\begin{array}{l}\text { Fibra dietética } \\
\text { soluble g/100 g }\end{array}$ & $\begin{array}{l}\text { Fibra dietética } \\
\text { insoluble g/100 g }\end{array}$ & $\begin{array}{l}\text { Relación } \\
\text { F.S./F.I. }\end{array}$ & Ref. \\
\hline Grateloupia turuturu & $48.1 \pm 1.0$ & $12.3 \pm 1.2$ & 3.9 & 71 \\
\hline Ulva clathrata & $21.9 \pm 0.9$ & $18.7 \pm 2.1$ & 1.2 & 72 \\
\hline Ulva lactuca & $27.2 \pm 1.2$ & $33.3 \pm 0.3$ & 0.8 & 33 \\
\hline Ulva lactuca & $20.53 \pm 0.28$ & $34.37 \pm 0.67$ & 0.6 & 73 \\
\hline Durvillaea antarctica (hojas) & $27.7 \pm 1.2$ & $43.7 \pm 0.3$ & 0.6 & 33 \\
\hline Durvillaea antarctica (tallo) & $24.2 \pm 2.5$ & $32.2 \pm 0.7$ & 0.8 & 33 \\
\hline Himanthalia elongate & $23.63 \pm 0.48$ & $13.51 \pm 0.45$ & 1.7 & 74 \\
\hline Laminaria saccharina & $17.12 \pm 0.84$ & $13.11 \pm 0.56$ & 1.3 & 74 \\
\hline
\end{tabular}


que se asemeja al síndrome metabólico humano, se comprobó que fucoxantina de algas pardas atenuó la ganancia de peso de tejido adiposo blanco, disminuyó la concentración de glucosa en la sangre y la insulina en el plasma (52).

Las algas también contienen polifenoles, compuestos bioactivos con alta capacidad antioxidante y también con actividad biológica específica que afecta la expresión de genes $(53,54)$. Existe gran interés científico por las propiedades de los polifenoles en la prevención de enfermedades relacionadas con el envejecimiento, enfermedades cardiovasculares y cáncer $(55,56)$. Las algas pardas contienen concentraciones más altas de polifenoles que algas rojas y verdes. La pared celular de las algas presenta una complejidad estructural y rigidez, está compuesta de una mezcla de polisacáridos ramificados y azufrados que se encuentran asociados con proteínas y iones, como calcio y potasio (42), lo que constituye el mayor obstáculo para la eficiente extracción de los constituyentes bioactivos intracelulares (57), para la determinación de polifenoles se debe recurrir a ensayos con diferentes solventes y tratamiento enzimático para una eficiente extracción $(42,58$, 59). La tabla 6 presenta la concentración de polifenoles en algas y en extractos de diversas algas.

Algas como ingredientes en alimentos

Conociendo los beneficios asociados al consumo de algas, éstas pueden ser usadas como un importante componente de la dieta; además de los beneficios nutricionales y saludables, las algas poseen características tecnológicas que les permiten ser incorporadas en alimentos, un ejemplo de ello son los productos cárnicos en base a emulsiones (60-65).

La incorporación de algas en productos cárnicos presenta muchos beneficios, por un lado, el sistema alga-carne posee proteínas de calidad, además las algas aportan compuestos antioxidantes, los cuales pueden mejorar la estabilidad oxidativa durante el almacenamiento del alimento (60). Debido a su composición, las algas tienen influencia en las propiedades del sistema gel/emulsión de la carne, favoreciendo la formación de estructuras más firmes y masticables, con mayor capacidad de retención de agua y grasa (66).

Lopez-López et al. (61) demostraron que la incorporación de algas en hamburguesas permite aumentar el contenido de fibra dietética y minerales como el calcio, y además mantener cantidades normales de sodio y una baja proporción de sodio/ potasio. López-López et al. (67), elaboraron hamburguesas con la incorporación de Undaria pinnatífida (alga parda conocida como wakame) y bajo contenido de sal. Se obtuvieron menores pérdidas de peso durante la descongelación, cocción y almacenamiento, relacionándose este comportamiento con el aporte de fibra dietética proveniente del alga ya que se forman estructuras más firmes, donde además se logra mejorar las propiedades emulsificantes, capacidad de retención de agua y materia grasa $(66,68)$. Las hamburguesas elaboradas

TABLA 5

Contenido de carotenoides y tocoles en algas

\begin{tabular}{|c|c|c|c|c|c|}
\hline Alga & $\begin{array}{l}\text { Carotenos } \\
\text { mg/kg b.s. }\end{array}$ & $\begin{array}{l}\text { Xantófilas } \\
\text { mg/kg b.s. }\end{array}$ & $\begin{array}{l}\beta \text {-caroteno } \\
\text { mg/kg b.s. }\end{array}$ & $\begin{array}{l}\text { Tocoles } \\
\mathrm{mg} / \mathrm{kg} \text { lípido }\end{array}$ & Ref. \\
\hline Ulva clathrata & $169.4 \pm 1.3$ & $10.2 \pm 5.2$ & - & - & 72 \\
\hline Ulva lactuca & - & - & - & $1071.4 \pm 9.2$ & 33 \\
\hline Durvillaea Antarctica (hojas) & - & - & - & $1112.9 \pm 8.2$ & 33 \\
\hline Durvillaea Antarctica (tallo) & - & - & - & $266.9 \pm 10.2$ & 33 \\
\hline Codium fragile & - & - & $197.9 \pm 1.8$ & $1617.6 \pm 10.3$ & 11 \\
\hline Gracilaria chilensis & - & - & $113.7 \pm 1.3$ & $391.9 \pm 9.7$ & 11 \\
\hline Macrocystis pyrifera & - & - & $17.4 \pm 1.0$ & $1457.2 \pm 11.4$ & 11 \\
\hline
\end{tabular}

\section{FIGURA 1}

Estructura de fucoxantina

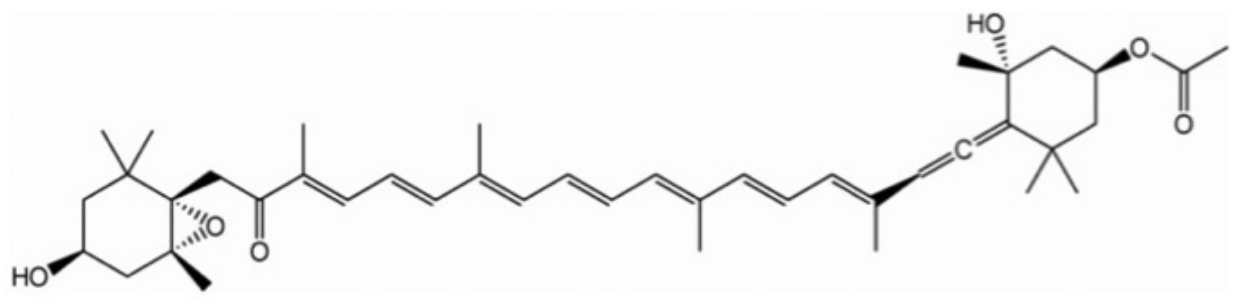


con adición de wakame presentaron textura más suave, mejorando la consistencia de la emulsión al modificar las propiedades reológicas de la fase continua de ésta; por otra parte las algas pardas presentan propiedades gelificantes debido a la presencia de iones de calcio y alginatos. No se obtuvo el mismo efecto con la incorporación de Himanthalia elongata (alga parda conocida como espagueti de mar) en salchichas bajas en sodio, se produjo reducción de la jugosidad, además se alteró el sabor, lo que redujo la aceptabilidad en general $(60,67)$. Jiménez-Colmenero et al. (62) incorporaron Himanthalia elongata en salchichas, las que fueron evaluadas sensorialmente con una escala no estructurada de 9 puntos, en la aceptabilidad de textura no existieron diferencias significativas entre la salchicha control (sin alga) y la con alga. En la aceptabilidad general, las salchichas con algas tuvieron una puntuación de 4.13 y 4.10 (con mayor y menor contenido de materia grasa respectivamente) y la salchicha control tuvo una puntuación significativamente más alta. Choi et al., (65) reemplazaron parcialmente la materia grasa en hamburguesas por Laminaria japonica (conocida como kombu), lo que provocó mayor elasticidad que la muestra control que contenía $20 \%$ de materia grasa. La evaluación sensorial, realizada con una escala descriptiva de 10 puntos, demostró que la incorporación de alga en concentraciones de 1 y $3 \%$ no variaba el flavor respecto a la muestra control, con puntuaciones de 8.1, 8.3 y 8.4 respectivamente. La calidad general mejoró significativamente con la incorporación de 1 y $3 \%$ de alga. Las hamburguesas con $5 \%$ de alga presentaron menor puntuación en las características sensoriales. La incorporación de algas en productos cárnicos permite mejorar la masticabilidad, reduciendo la elasticidad y cohesividad (66).

Las algas también se pueden incorporar en pastas con buenos resultados. Prabhasankar et al. (69) desarrollaron pastas con 1, 2.5 y $5 \%$ de Sargassum marginatum, un alga parda de la India. La incorporación del alga mejoró el comportamiento del gluten, sin embargo no hubo efecto sobre las propiedades antioxidantes de la pasta como se esperaba. La incorporación de Undaria pinnatífida o wakame en semolina para la preparación de pastas dio muy buenos resultados. Se produjo un aumento en el contenido de proteínas, materia grasa y fibra dietética, además de fucoxantina y fucosterol en las pastas elaboradas con wakame como ingrediente. Se puede llegar hasta niveles de 10\% de incorporación del alga, ya que se logra alta aceptabilidad en análisis sensorial, sin embargo la incorporación de 20 o 30\% de alga disminuye la aceptabilidad, afectando el sabor, apariencia y sensación bucal (70).

\section{CONCLUSIÓN}

La calidad nutritiva de algas marinas junto al alto contenido de compuestos bioactivos con efecto saludable, son dos razones importantes para aumentar su consumo. Además, las algas poseen propiedades tecnológicas propias de estructuras proteicas lo que permite su incorporación en alimentos cárnicos y en pastas, manteniendo o mejorando su calidad sensorial, nutritiva y saludable.

\section{RESUMEN}

Las algas marinas se han consumido en Asia desde tiempos remotos, mientras que en países occidentales su principal aplicación ha sido como agente gelificante y coloide para la industria de alimentos, farmacéutica y cosmética. Las algas son buena fuente de nutrientes como proteínas, vitaminas, minerales y fibra dietética, al respecto, la fibra dietética de algas es particularmente rica en fracción soluble. Si se comparan las algas con vegetales terrestres, se encuentran más componentes beneficiosos para la salud, como ácidos grasos $\omega-3$ y moléculas bioactivas. Las algas sintetizan diversos metabolitos secundarios que presentan actividad antioxidante, antiinflamatoria, anticancerígena y antidiabética. Por lo tanto, las algas se pueden considerar una fuente natural de gran interés ya que contienen compuestos con numerosas actividades biológicas y pueden ser usadas como ingrediente funcional en muchas aplicaciones industriales como en alimentos funcionales.

Palabras clave: algas, fibra dietética, polifenoles, pigmentos.

\section{BIBLIOGRAFÍA}

1. Santelices B. Algas marinas de Chile. Ediciones Universidad Católica de Chile. Santiago, Chile. 1989.

2. Swamy M.L.A. Marine Algal Sources for Treating Bacterial Diseases. Adv Food Nut Res 2011; 64: 71-84.

3. Rajapakse N, Kim S. Nutritional and digestive health benefits of seaweeds. Adv Food Nut Res 2011; 64: 17-28.

4. Teas, J., Pino, S., Critchley, A., and Braverman, L. E. Variability of iodine content in common commercially available edible seaweeds. Thyroid 2004; 14: 836-41.

5. Gómez-Gutierrez C., Guerra-Rivas G., Soria-Mercadol., Ayala-Sánchez N. Marine Edible Algae as Disease Preventers. Adv Food Nut Res 2011; 64: 29-39.

6. Dhargalkar V.K., Verlecar X.N. Southern Ocean seaweeds:

\section{TABLA 6}

Contenido de polifenoles totals en algas y extractos de algas

$\begin{array}{lll}\text { Alga } & \text { Polifenoles totales (mg AGE/100 g) } & \text { Ref. } \\ \text { Stypocaulon scoparium (extracto acuoso) } & 328.7 \pm 2.87 \text { (mg AGE/100 g alga seca) } \\ \text { Stypocaulon scoparium (extracto metanólico) } & 255.2 \pm 1.59 \text { (mg AGE/100 g alga seca) } \\ \text { Ulva lactuca } & 2.86 \pm 0.04(\mathrm{mg} \mathrm{AGE/100} \mathrm{g} \mathrm{alga} \mathrm{seca)} & 58 \\ \text { Alaria esculenta } & 2.80 \pm 0.05(\mathrm{mg} \mathrm{AGE/100} \mathrm{g} \mathrm{alga} \mathrm{seca)} \\ \text { Turbinaria conoides (extracto metanólico) } & 1.231 \pm 0.173 \text { (mg AGE/g alga) } & 75 \\ \text { Turbinaria conoides (extracto en éter etílico) } & 1.190 \pm 0.006 \text { (mg AGE/g alga) } & 75 \\ \text { Sargassum marginatum (extracto acuoso) } & 0.29 \text { (mg AGE/100 g alga seca) } & 59 \\ \text { Turbinaría conoides (extracto acuoso) } & 0.86 \text { (mg AGE/100 g alga seca) } \\ \text { Gracilaria birdiae (extracto etanólico) } & 1.13 \pm 0.03 \text { (mg AGE/100 g extracto) } & 39 \\ \text { Gracilaria birdiae (extracto metanólico) } & 1.06 \pm 0.07 \text { (mg AGE/100 g extracto) }\end{array}$


A resource for exploration in food and drugs. Aquaculture 2009; 287: 229-242

7. Lahaye M. (1991). Marine algae as sources of fibres: determination of soluble and insoluble DF contents in some sea vegetables. I Sci Food Agr 54: 587-94.

8. Watanabe 7 Nakano Tamura Yamanaka $H$ Vitamin B12 metabolism in a photosynthesizing green alga, Chlamydomonas reinhardtii. Biochim Biophys Acta 1991; 1075:36-41.

9. Jimenez-Escrig \& Goñi C. Nutritional evaluation and physiological effects of edible seaweeds. Arch Latinoam Nutr 1999; 49: 114-20.

10. Wong K.H. Cheung P. Nutritional evaluation of some subtropical red and green seaweeds Part II. In vitro protein digestibility and amino acid profiles of protein concentrates. Food Chem 2001; 72:11-7.

11. Ortiz J, Urquiche E, Robert P, Romero N, Quitral V, Llantén C. Functional and nutritional value of the chilean seaweeds Codium fragile, Gracilaria chilensis and Macrocystis pyrifera. Eur J Lipid Sci Technol 2009; 111: 320 - 7.

12. Aruoma O.I, Halliwell B, Hoey B.M, Butler J. The antioxidant action of taurine, hypotaurine and their metabolic precursors. Biochem J 1988; 256: 251-5.

13. Huxtable R J. Physiological actions of taurine. Physiol Rev 1992; 72:101-63.

14. Schaffer S, Takahashi K, Azuma A. Role of osmoregulation in the actions of taurine. Amino Acids 2000; 19: $527-46$.

15. Wójcik OA Koenig KL Zeleniuch-Jacquotte A, Costa M Chen $y$ The potential protective effects of taurine on coronary heart disease. Atherosclerosis 2010; 208:19-25.

16. Larsen R., Eilertsen K., Elvevoll E. Health benefits of marine foods and ingredients, Biotechnol Adv 2011; 29: 508-18.

17. Gaull G.F. Taurine in the nutrition of the human infant. Acta Paediatr 1982; 71: 38 - 40

18. Chesney R. Taurine: is required for infant nutrition. J Nut 1988; 118: 6 - 10.

19. Cañas P. Biological and nutritional role of taurine and its derivatives. Rev Chil Nutr 2002; 29: 286 - 92.

20. Dawczynski C, Schubert R., Jahreis G. Amino acids, fatty acids and dietary fibre in edible seaweed products. Food Chem 2007; 103: 891-9.

21. Matanjun, P., Mohamed, S., Mustapha, N. M., \& Muhammad, K. Nutrient content of tropical edible seaweeds, Eucheuma cottonii, Caulerpa lentillifera and Sargassum polycystum. J Appl Phycol 2009; 21: 1-6.

22. Kumar M., Gupta V., Kumari P. Reddy C., Jha B. Assessment of nutrient composition and antioxidant potential of Caulerpaceae seaweeds. J Food Compost Anal 2011; 24:270-8

23. Mohamed S, Hashim S N, Abdul H. Seaweeds: a sustainable functional food for complementary and alternative therapy. Trends Food Sci Technol 2012; 23: 83-96.

24. Lopez-Huertas E. Health effects of oleic acid and long chain omega-3 fatty acids (EPA and DHA) enriched milks. A review of intervention studies Pharmacol Res 2010; 61:200-7.

25. Urbano M G, Goñi I. Bioavailability of nutrients in rats fed on edible seaweeds, Nori (Porphyra tenera) and Wakame (Undaria pinnatifida), as a source of dietary fibre. Food Chem 2002; 76: 281-6.

26. Sirot $V$, Dumas $C$, Desquilbet $L$, Mariotti $F$, Legrand $P$, Catheline D, Leblanc J C, Margaritis I. A restricted cubic spline approach to assess the association between high fat fish intake and red blood cell EPA + DHA content. Nutr
Metab Cardiovasc Dis 2012; 22:318-26.

27. Kris-Etherton P M, Grieger J A, Etherton $T$ D. Dietary reference intakes for DHA and EPA. Prostag Leukotr Ess 2009; 81: 99 - 104.

28. Hibbeln J $R$, Nieminen $L R$, Blasbalg $T L$, Riggs $J A$, Lands $W E$. Healthy intakes of $n-3$ and $n-6$ fatty acids: estimations considering worldwide diversity. Am J Clin Nutr 2006; 83: $1483-93$.

29. Simopoulos A P. The importance of the ratio of omega-6/ omega-3 essential fatty acids. Biomed Pharmacoth 2002; 56: $365-79$.

30. Fleurence J, Gutbier G, Mabeau S, Leray C. Fatty acids from 11 marine macroalgae of the French Brittany coast. J Appl Phycol 1994; 6: 527-32.

31. Kumari P, Kumar M, Gupta V, Reddy C.R.K, Jha B. Tropical marine macroalgae as potential sources of nutritionally important PUFAs. Food Chem 2010; 120: 749-57.

32. Rasmussen R.S., Morrisey M. Marine biotechnology for production of food ingredients. Adv Food Nutr Res 2007; 52: 237-92.

33. Ortiz J, Romero $N$, Robert $P$, Araya J, Lopez-Hernández J, Bozzo C, Navarrete E, Osorio A, Rios A. Dietary fiber, amino acid, fatty acid and tocopherol contents of the edible seaweeds Ulva lactuca and Durvillaea antárctica. Food Chem 2006; 99 : 98-104.

34. Pak N. Fibra dietética en verduras cultivadas en Chile. Arch Latinoam Nutr 2000; 50: 97-101.

35. Pak N. Fibra dietética en frutas cultivadas en Chile. Arch Latinoam Nutr 2003; 53: 413-7.

36. Pak N, Araya H. Macroalgas comestibles de Chile como fuente de fibra dietética: Efecto de la digestibilidad aparente de proteínas, fibra, energía y peso de deposiciones en ratas. Arch Latinoam Nutr 1996; 46: 42 - 6.

37. Elleuch M, Bedigian D, Roiseux O, Besbes S, Blecker C, Attia $H$. Dietary fibre and fibre-rich by-products of food processing: Characterization, technological functionality and commercial applications: A review. Food Chem 2011; 124: $411-21$.

38. Chandini $S, K$, Suresh P. V, Bhaskar N. Seaweeds as source of nutritionally beneficial compounds - A review. J Food Sci Technol 2008; 45: 1 - 13.

39. Wang $T$, Jónsdóttir $R$, Kristinsson $H G$, Hreggvidsson $G O$, Jónsson J, Thorkelsson G, Ólafsdóttir G. Enzyme-enhanced extraction of antioxidant ingredients from red algae Palmaria palmate. LWT - Food Sci Technol 2010; 43: 1387-93.

40. Souza B, Cerqueira M A, Martins J T, Quintas M A C, Ferreira $A$ C S, Teixeira J A, Vicente $A$ A. Antioxidant potential of two red seaweeds from brazilian coasts. J Agric Food Chem 2011; 59: 5589 - 94.

41. Cho M, Lee H, Kang I, Wond M, You S. Antioxidant properties of extract and fractions from Enteromorpha prolifera, a type of green seaweed. Food Chem 2011; 127: 999-1006.

42. Wijesinghe W. A.J.P., Jeon Y-J. Enzyme-assistant extraction (EAE) of bioactive components: A useful approach for recovery of industrially important metabolites from seaweeds: A review. Fitoterapia 2012; 83: 6-12.

43. Pangestuti R, Kim S. Biological activities and health benefit effects of natural pigments derived from marine algae. J Funct Foods 2011; 3:255-66.

44. Bohn T. Bioavailability of non-provitamin A carotenoids. Curr Nutr Food Sci 2008; 4:240-58.

45. Okuzumi, J., Nishino, H., Murakoshi, M., Iwashima, A., Tanaka, Y., Yamane, T., Fujita, Y., and Takahashi, T. Inhibitory effects of fucoxanthin, a natural carotenoid, on Nmyc 
expression and cell cycle progression in human malignant tumor cells. Cancer Lett 1990; 55: 75-81.

46. Sasaki, K., Ishihara, K., Oyamada, C., Sato, A., Fukushi, A., Arakane, T., Motoyama, M., Yamazaki, M., and Mitsumoto, $M$. Effects of fucoxanthin addition to ground chicken breast meat on lipid and colour stability during chilled storage, before and after cooking. Asian-Aust J Anim Sci 2008; 21:1067-72.

47. Maeda H, HosokawaM, Sashima T, Miyashita K. Chapter 32 Antiobesity Effect of Fucoxanthin from Edible Seaweeds and Its Multibiological Functions. In "Functional Food Health", T. Shibamoto, K. Kanazawa, F. Shahidi, and C. T. Ho, Eds, ACS Publications, Washington. 2008. pp. 376-88.

48. Heo $S J$, Yoon $W J$, Kim $K N$, Ahn $G N$, Kang S M, Kang $D H$, Affan A, Oh C, Jung W. K, Jeon Y J. Evaluation of anti-inflammatory effect of fucoxanthin isolated from brown algae in lipopolysaccharide-stimulated RAW 264.7 macrophages. Food Chem Toxicol 2010; 48: 2045-51.

49. Das S K, Ren R, Hashimoto T, Kanazawa K. Fucoxanthin induces apoptosis in osteoclast-like cells differentiated from RAW264. 7 cells. J Agric Food Chem 2010; 58:6090-5.

50. Shimoda H, Tanaka, Shan S, Maoka T. Anti-pigmentary activity of fucoxanthin and its influence on skin mRNA expression of melanogenic molecules. J Pharm Pharmacol 2010; 62:1137-45.

51. Hayato, M., Masashi, H., Tokutake, S., Nobuyuki, T., Teruo, K., \& Kazuo, M. Fucoxanthin and its metabolite, fucoxanthinol, suppress adipocyte differentiation in 3T3-L1 cells. Int J Mol Med 2006; 18: 147-52.

52. Maeda H., Hosokawa M., Sashima T., Miyashita K., Dietary Combination of Fucoxanthin and Fish Oil Attenuates the Weight Gain of White Adipose Tissue and Decreases Blood Glucose in Obese/Diabetic KK-Ay Mice. J Agric Food Chem 2007; 55: 7701-6.

53. Wang $T$, Jónsdóttir $R$, Ólafsdóttir $G$. Total phenolic compounds, radical scavenging and metal chelation of extracts from Icelandic seaweeds. Food Chem 2009; 116:240-8.

54. Rodrigo R., Miranda A., Vergara L. Modulation of endogenous antioxidant system by wine polyphenols in human disease Clinica Chimica Acta 2011; 412: 410-24.

55. Keyrouz R., Abasq M.L., Le Bourvellec C., Blanc N., Audibert L., ArGall E., Hauchard D. Total phenolic contents, radical scavenging and cyclic voltammetry of seaweeds from Brittany. Food Chem 2011; 126 : 831-6.

56. Stagos D., Amoutzias G., Matakos A., Spyrou A., Tsatsakis A., Kouretas D. Chemoprevention of liver cancer by plant polyphenols. Food Chem Toxicol 2012; 50: 2155-70.

57. Deniaud E., Quemenerb B., Fleurence J., Lahaye M. Structural studies of the mix-linked $\beta-(1$ ? 3)/ $\beta$-(1 [? 4)-d-xylans from the cell wall of Palmaria palmata (Rhodophyta). Int J Biol Macromol 2003; 33: 9-18.

58. López A., Rico M., Rivero A., Suárez de Tangil M. The effects of solvents on the phenolic contents and antioxidant activity of Stypocaulon scoparium algae extracts. Food Chem 2011; 125:1104-9

59. Devi G.K., Manivannan K., Thirumaran G., Rajathi A.A., Anantharaman P. In vitro antioxidant activities of selected seaweeds from Southeast coast of India Asian Pac J Trop Med 2011; 4: 205-11.

60. López-López I, Bastida S, Ruiz-Capillas C, Bravo L, Larrea M T, Sánchez-Muniz F, Cofrades S, Jiménez-Colmenero F. Composition and antioxidant capacity of low-salt meat emulsion model systems containing edible seaweeds. Meat
Sci 2009; 83: $492-8$.

61. López- López I, Cofrades S, Ruiz-Capillas C, JiménezColmenero F. Design and nutritional properties of potential functional frankfurters base on lipid formulation, added seaweed and low salt content. Meat Sci 2009;83: 255-62.

62. Jimenez-Colmenero F, Cofrades S, López-López I, RuizCapillas C, Pintado T, Solas M T. Technological and sensory characteristics of reduced/low-fat, low-salt frankfurters as affected by the addition of konjac and seaweed. Meat Sci 2010; 84: 356-3.

63. Weiss J, Gibis M, Schuh V, Salminen H. Advances in ingredient and processing systems for meat and meat products. Meat Sci 2010; 86: 196-213.

64. Mendis E, Kim S. Present and Future Prospects of Seaweeds in Developing Functional Foods. Adv Food Nutr Res 2011; 64: 1-15

65. Choi Y.S, Han D.J, Kim H.Y, Kim H.W. Effects of Laminaria janponica on the physico-chemical and sensory characteristics of reduced-fat pork patties. Meat Sci 2012; 91:1-7.

66. Cofrades S, López-lópez I, Solas M.T, Bravo L, Jiménezcolmenero $F$. Influence of different types and proportions of added edible seaweeds on characteristics of low-salt gel/ emulsion meat systems. Meat Sci 2008; 79: $767-76$.

67. López-López I, Cofrades S, Yakan A, Solas M T, JiménezColmenero F. Frozen storage characteristics of low-salt and low-fat, beef patties as affected by Wakame addition and replacing pork backfat olive oil-in-water emulsion. Food Res Int 2010; 43: $1244-54$.

68. López-López I, Cofrades S, Jiménez-Colmenero F. Low-fat frankfurters enriched with n-3 PUFA and edible seaweed: Effects of olive oil and chilled storage on physicochemical, sensory and microbial characteristics. Meat Sci 2009; 83: $148-54$.

69. Prabhasankar, P., Ganesan, P., \& Bhaskar, N. Influence of Indian brown seaweed (Sargassum marginatum) as an ingredient on quality, biofunctional and microstructure characteristics of pasta. Food Science and Technology International. Food Sci Tech Int 2009; 15: 471-9.

70. Prabhasankar, P., Ganesan, P., Bhaskar, N., Hirose, A., Stephen, N., Gowda, L. R., Hosokawa M., Miyashita K. Edible Japanese seaweed, wakame (Undaria pinnatifida) as an ingredient in pasta: Chemical, functional and structural evaluation. Food Chem 2009; 115: 501-8.

71. Denis C., Morançais M., Li M., Deniaud E., Gaudin P., Wielgosz-Collin G., Barnathan G., Jaouen P., Fleurence J. Study of the chemical composition of edible red macroalgae Grateloupia turuturu from Brittany (France). Food Chem 2010; 119: 913-7.

72. Peña-Rodriguez A., Mawhinney T., Ricque-Marie D., CruzSuárez E. Chemical composition of cultivated seaweed Ulva clathrata (Roth) C. Agardh. Food Chem 2011; 129:491-8.

73. Yaich H., Garna H., Besbes S., Paquot M., Blecker C., Attia $H$. Chemical composition and functional properties of Ulva lactuca seaweed collected in Tunisia. Food Chem 2011; 128: 895-901.

74. Gómez-Ordóñez E., Jiménez-Escrig A., Rupérez P. Dietary fibre and physicochemical properties of several edible seaweeds from the northwestern Spanish coast. Food Res Int 2010; 43: 2289-94.

75. Nwosu F., Morris J., Lund V.A., Stewart D., Ross H.A., McDougall G.J. Anti-proliferative and potential anti-diabetic effects of phenolic-rich extracts from edible marine algae. Food Chem 2011; 126:1006-12. 